\title{
Short communication: A nanoemulsified form of oil blends positively affects the fatty acid proportion in ruminal batch cultures
}

\author{
M. El-Sherbiny, ${ }^{\star} †$ A. Cieslak, ${ }^{*}$ E. Pers-Kamczyc,‡ J. Szczechowiak, ${ }^{*}$ D. Kowalczyk, ${ }^{\star}$ \\ and M. Szumacher-Strabel ${ }^{* 1}$ \\ ${ }^{*}$ Department of Animal Nutrition and Feed Management, Poznan University of Life Sciences, 60637 Poznan, Poland \\ †Department of Dairy Sciences, National Research Centre, 12622 Giza, Egypt \\ łlnstitute of Dendrology, Polish Academy of Sciences, 62035 Kornik, Poland
}

\section{ABSTRACT}

Two consecutive rumen batch cultures were used to study the effect of nanoemulsified oils as a new type of supplement, on the in vitro fatty acid proportion and vaccenic acid formation. Three levels $(3,5$, and $7 \%$ ) of 2 different oil blends [soybean:fish oil (SF) or rapeseed-fish oil (RF)] were used. Both oil blends were used either in the raw form (SF or RF, respectively) or in the nanoemulsified form (NSF or NRF, respectively). The diets were the control $(0 \%)$, which consisted of a dry total mixed ration without any supplements, the control plus 3, 5, or $7 \%$ of the SF or RF oil blend in appropriate form (raw or nanoemulsified). For each treatment, 6 incubation vessels were used. Each batch culture was incubated for $24 \mathrm{~h}$ and conducted twice in 2 consecutive days. All supplements were calculated as a percentage of the substrate dry matter $(400 \mathrm{mg})$. Nanoemulsified supplements were recalculated to make sure the oil amount was equal to the raw oil supplementation levels. The results from both experiments indicated that the proportions of vaccenic acid and cis-9,trans-11 C18:2 increased when a raw oil blend was supplemented; on the other hand, no influence of nanoemulsified form of oil blend was observed on the proportion cis-9,trans-11 C18:2. Generally, supplementation with the nanoemulsified oil blends had less effect on biohydrogenation intermediates than the raw form of oil blends. However, the nanoemulsified form had a greater effect on the increase of n-3 and n- 6 fatty acids. Nanoemulsified oil blends had a positive effect on decreasing the transformation rate of polyunsaturated fatty acids to saturated fatty acids in the biohydrogenation environment. Supplements of nanoemulsified oil blends tended to be more effective than supplements of

Received January 9, 2015.

Accepted September 15, 2015.

${ }^{1}$ Corresponding author: mstrabel@up.poznan.pl raw oils in preserving a greater proportion of polyunsaturated fatty acids in the fermentation culture.

Key words: rumen, batch culture, nanoemulsion, oil blend, unsaturated fatty acid

\section{Short Communication}

For decades, various feed supplements have been introduced to moderate rumen fatty acid proportions and consequently to modify ruminant products. There is considerable interest in developing nutritional strategies to boost the UFA of milk and meat. The UFA offer potential benefits for human health, mainly attributed to CLA isomers and to n- 3 and n- 6 fatty acids. Increasing the supply of UFA in the human diet can help to prevent or delay atherosclerosis and coronary heart disease, while helping to avoid inflammatory conditions. Increasing the supply of UFA can also slow the growth of tumor cells (McCrorie et al., 2011; Koba and Yanagita, 2014). Some of the most commonly used forms of UFA supplementation are edible oils of plant or marine origin. Oils show an ability to modulate the rumen fatty acid proportion by affecting the activity of rumen microorganisms, especially the bacterial population (AbuGhazaleh and Ishlak, 2014; Boerman and Lock, 2014). Supplements do have some limits and restrictions in ruminant nutrition because of the possible negative effect on rumen fermentation (Martínez Marín et al., 2013; Ishlak et al., 2014). These limits make it desirable to find other dietary UFA sources that can affect and modulate the rumen fatty acid proportion without negatively affecting microorganisms or rumen fermentation indicators.

Recently, nanotechnology has found innumerable applications in different lines of work. Delivery of bioactive components using nanoscale technology has been documented in pharmaceutics as well as the cosmetic and food sciences (Fathi et al., 2012; Ghosh et al., 2014; Zhang et al., 2014). Nanoemulsion is one of the most important nanotechnology applications, with wide usage 
in several scientific and practical fields. A nanoemulsion is defined as a multiphase colloidal dispersion formed by a mixture of one liquid that is dispersed as nanoscale droplets in another immiscible liquid. Physical shareinduced rupturing takes place, leading to a droplet diameters of $<100 \mathrm{~nm}$ (Mason et al., 2006). The present preliminary experiments established new approaches for modulating the rumen fatty acid proportion by incorporating an oil-in-water nanoemulsion as a novel dietary component. The effect of incorporating an oil-inwater nanoemulsion into ruminant diets has never been investigated. We hypothesized that nanoscale droplets of oil blends rich in PUFA, added directly to the rumen fermentation culture, would inhibit microbial reactions; that is, lipolysis and biohydrogenation. This inhibition could prevent more UFA from being lost during those processes. Therefore, the main objective of the present study was to evaluate the effect of nanoemulsified oil blends rich in PUFA on rumen fatty acid proportions after $24 \mathrm{~h}$ of batch incubation.

Three hours after the morning feeding, the rumen inoculum was obtained from 3 ruminally cannulated Polish Holstein-Friesian dairy cows that had an average BW of $600 \mathrm{~kg}$. The ruminal content was collected from the top, bottom, and middle of the rumen of each cow separately. The ruminal contents from all 3 cows were equally blended and then strained through 4 layers of cheesecloth into a Schott Duran bottle (Schott North America Inc., Elmsford, NY), and immediately transported to the laboratory in a water bath heated to $39^{\circ} \mathrm{C}$. Two separate experimental trials on 2 consecutive days were carried out in the batch culture system according to the modified protocol of Szumacher-Strabel et al. (2004). Each in vitro run began with a single fresh collection of rumen fluid.

Briefly, rumen fluid was diluted with a buffer solution (292 mg of $\mathrm{K}_{2} \mathrm{HPO}_{4} \cdot 3 \mathrm{H}_{2} \mathrm{O}, 240 \mathrm{mg}$ of $\mathrm{KH}_{2} \mathrm{PO}_{4}, 480 \mathrm{mg}$ of $\left(\mathrm{NH}_{4}\right)_{2} \mathrm{SO}_{4}, 480 \mathrm{mg}$ of $\mathrm{NaCl}, 100 \mathrm{mg}$ of $\mathrm{MgSO}_{4} \cdot 7 \mathrm{H}_{2} \mathrm{O}$, $64 \mathrm{mg}$ of $\mathrm{CaCl}_{2} \cdot 2 \mathrm{H}_{2} \mathrm{O}, 4 \mathrm{mg}$ of $\mathrm{Na}_{2} \mathrm{CO}_{3}$, and $600 \mathrm{mg}$ of cysteine hydrochloride per liter) at a ratio of 1:4. Then, a portion of this blend $(40 \mathrm{~mL})$ was put into 125 -mL glass incubation vessels that already contained $400 \mathrm{mg}$ of dried substrate (the TMR). The substrate used in our experiments was similar to the diet offered to the ruminally cannulated dairy cows (rumen fluid donors). All ingredients were dried first and then each dried ingredient was milled separately. A homogeneous mixture of the experimental substrate was made on a DM basis by mixing together the following amounts of the dried ingredients: maize silage (396 $\mathrm{g} / \mathrm{kg}$ of DM), lucerne silage $(71 \mathrm{~g} / \mathrm{kg}$ of DM), grass silage $(104 \mathrm{~g} / \mathrm{kg}$ of DM), beet pulp (113 g/ kg of DM), brewer's grain $(85 \mathrm{~g} / \mathrm{kg}$ of DM), extracted rapeseed meal $(42 \mathrm{~g} / \mathrm{kg}$ of DM), commercial concentrate containing $18 \% \mathrm{CP}$ $(185 \mathrm{~g} / \mathrm{kg}$ of DM), and a mineral mixture $(4 \mathrm{~g} / \mathrm{kg}$ of DM). Subsequently, $400 \mathrm{~g}$ of the dried feed mixture was added to each bottle. The bottles were incubated for 6 $\mathrm{h}$ at $39^{\circ} \mathrm{C}$ before the start of the experiment.

Three different doses of 2 different oil blends, in raw and nanoemulsified forms, were evaluated as supplements in 2 consecutive, short-term in vitro batch fermentation trials. The doses of the raw oil blends (3, 5 , and $7 \%$ ) were calculated based on the substrate's DM. The raw oil supplements were then added on top of the $400 \mathrm{~g}$ of TMR. The oil contents of the prepared nanoemulsified form were equal to the oil doses used in the raw oil-blend treatments $(3,5$, and $7 \%)$. The amount of the supplemented nanoemulsions, however, were recalculated based on the oils used in the nanoemulsion preparation (about 15\% of the oil blends) to be about 20,33 , and $47 \%$ on a substrate DM basis. The nanoemulsified oil blends were then added directly to the bottle containing both the buffered rumen fluid and substrate.

Two different oil blends [a 1:1 soybean oil:fish oil blend (SF) and a 1:1 rapeseed oil:fish oil blend (RF)] in 2 forms [raw (SF and RF) and nanoemulsified (NSF and NRF)] were used during each experimental batch culture. We analyzed 3 levels $(3,5$, and $7 \%)$ for each type of oil blend and included a control (0\%), which consisted of TMR without any supplement. Each batch culture trial was repeated twice on 2 days with 2 different incubation fluids. Six incubation vessels (bottles) were used in each treatment. Six more vessels contained the control substrate (the dry TMR), and another 6 blank vessels contained culture fluid without the substrate. The bottles were filled with $\mathrm{CO}_{2}$, closed with a rubber stopper, and sealed with aluminum. Then, the bottles were incubated for $24 \mathrm{~h}$ in anaerobic conditions at a $\mathrm{pH}$ was 6.5 and a temperature of $39^{\circ} \mathrm{C}$. The bottles were agitated every 30 min while being incubated. The oil-in-water nanoemulsion was prepared using a Hielscher UP50H ultrasonic processor (80\% amplitude for 20 min; Hielscher Ultrasonics, Teltow, Germany) as described by Ahmadi Lakalayeh et al. (2012). Rapeseed oil, soybean oil, and fish oil were used as the inner phase. The only surfactant used was Tween 80 (SigmaAldrich, Poznan, Poland). The oil-in-water emulsion formulation was composed of $15 \%$ oil, $5.6 \%$ Tween 80 , and $79.4 \%$ deionized water as suggested by Kentish et al. (2008).

Fatty acid methyl esters in the TMR, oils, nanoemulsions, and fermentation fluid samples were extracted and analyzed after $24 \mathrm{~h}$ of incubation according to Cieslak et al. (2009) with some modification. Briefly, $2,500 \mu \mathrm{L}$ of rumen fluid was suspended in $3 \mathrm{~mL}$ of $2 M$ 
$\mathrm{NaOH}$ and incubated in a block heater at $90^{\circ} \mathrm{C}$ for 40 min. After cooling to room temperature, $1.7 \mathrm{~mL}$ of 4 $M \mathrm{HCl}$ was added to lower the $\mathrm{pH}$ to below 2 . Before extraction, $2 \mathrm{~mL}$ of distilled diethyl ether was added to each sample. The tubes were shaken vigorously for $10 \mathrm{~min}$, and then centrifuged at $6,160 \times g$ for 1 min at $20^{\circ} \mathrm{C}$. The extraction procedure was repeated 3 times, and the supernatant was finally evaporated at $30^{\circ} \mathrm{C}$ for 10 min under a flux of nitrogen using a Techne DriBlock heater model DB-3 (Bibby Scientific Ltd., Staffordshire, UK). The extracted fatty acids were esterified using $0.5 \mathrm{M} \mathrm{NaOH}$ in methanol and subsequently converted to FAME using boron trifluoride (Fluka, Sigma Aldrich). Then, $0.34 \mathrm{M} \mathrm{NaCl}$ and hexane were added and the mixture was shaken vigorously. The organic phase containing the FAME was used for GC analyses using a Varian Star CP 3800 (Agilent Technologies, Santa Clara, CA) fitted with a flame-ionization detector and a 100-m fused-silica capillary column (internal diameter of $0.25 \mathrm{~mm}$ ) coated with $0.2 \mu \mathrm{m}$ of CP-Sil 88 (Chrompack, Varian). The bacteria were counted under a light microscope $(400 \times$ magnification) in a Thoma chamber (0.02 mm depth, Blau Brand, Wertheim, Germany), according to Ericsson et al. (2000). In vitro DM digestibility (IVDMD) was analyzed according to the procedure described by Cieslak et al. (2014); briefly, a parallel trial with the same experimental design and bottle numbers as for the previously described batch culture trials was used for IVDMD estimation; $40 \mathrm{~mL}$ of buffered rumen fluid was incubated with $400 \pm 1 \mathrm{mg}$ of substrate and the proper supplementation for $24 \mathrm{~h}$ at $39^{\circ} \mathrm{C}$. After incubation, the content of the incubation flasks was transferred to previously weighed crucibles. The residues of incubation were washed with $50 \mathrm{~mL}$ distilled water and dried at $105^{\circ} \mathrm{C}$ for $3 \mathrm{~d}$. The percentage loss in weight of the feed DM was determined and presented as IVDMD.

Statistical analysis was completed using 3-way ANOVA with the Tukey post hoc test. The main effects of oil (nanoemulsified vs. raw), level of supplementation $(0,3,5$, and $7 \%)$, repetition of the experiments, and the interaction between type and supplementation level were determined. However, we found no effect of experiment repetition on the obtained results; therefore, it was excluded from the model and all data were re-run using 2-way ANOVA. Polynomial contrasts were used to describe linear and quadratic responses to the level of supplementation for all oil types. In all analyses, significant effects were declared at $P<0.05$. All analyses were performed using SAS software (version 9.3; SAS Institute Inc., Cary, NC).

The fatty acid proportions of the TMR, nanoemulsified oil blends, and raw oil blends are presented in Table 1. The fatty acid composition of nanoemulsions prepared for both batch culture experiments was slightly affected by the sonication process. This effect explains

Table 1. Fatty acid proportion in TMR (main substrate), nanoemulsion, and oils mix (g/100 g of fatty acids)

\begin{tabular}{|c|c|c|c|c|c|}
\hline \multirow[b]{2}{*}{ Item } & \multirow[b]{2}{*}{ TMR } & \multicolumn{4}{|c|}{ Supplement ${ }^{1}$} \\
\hline & & $\mathrm{SF}$ & $\mathrm{NSF}$ & $\mathrm{RF}$ & NRF \\
\hline C14:0 & 0.62 & 1.47 & 1.44 & 1.27 & 1.13 \\
\hline C16:0 & 16.6 & 9.88 & 9.52 & 6.66 & 6.66 \\
\hline C18:0 & 3.24 & 4.13 & 3.90 & 2.13 & 2.40 \\
\hline cis-9 C18:1 & 22.3 & 30.8 & 34.9 & 49.1 & 51.7 \\
\hline cis-9,cis-12 C18:2 & 40.5 & 30.9 & 27.5 & 16.3 & 15.1 \\
\hline cis-9,cis-12,cis-15 C18:3 & 6.48 & 6.20 & 5.50 & 6.90 & 6.18 \\
\hline $\mathrm{C} 20: 4 \mathrm{n}-6$ & $\mathrm{ND}^{2}$ & 1.72 & 1.50 & 1.71 & 1.44 \\
\hline C20:5n-3 & ND & 0.19 & 0.15 & 0.15 & 0.12 \\
\hline $\mathrm{C} 22: 6 \mathrm{n}-3$ & ND & 2.36 & 2.24 & 2.35 & 2.12 \\
\hline$\Sigma \mathrm{SFA}^{3}$ & 23.5 & 16.9 & 16.6 & 11.2 & 11.5 \\
\hline$\Sigma \mathrm{UFA}^{4}$ & 76.5 & 83.1 & 83.4 & 88.8 & 88.5 \\
\hline$\Sigma \mathrm{PUFA}^{5}$ & 49.1 & 43.7 & 42.6 & 33.3 & 30.5 \\
\hline$\Sigma_{\text {MUFA }}{ }^{6}$ & 27.4 & 39.5 & 40.8 & 55.4 & 57.9 \\
\hline
\end{tabular}

${ }^{1}$ Supplements: NSF $=1: 1$ blend of nanoemulsified soybean oil:fish oil; NRF = 1:1 blend of nanoemulsified rapeseed oil:fish oil; $\mathrm{SF}=1: 1$ blend of soybean oil:fish oil; $\mathrm{RF}=1: 1$ blend of rapeseed oil:fish oil.

${ }^{2} \mathrm{ND}=$ not detected.

${ }^{3} \mathrm{Sum}=\mathrm{C} 6, \mathrm{C} 8, \mathrm{C} 10, \mathrm{C} 12, \mathrm{C} 14, \mathrm{C} 15, \mathrm{C} 16, \mathrm{C} 17, \mathrm{C} 18, \mathrm{C} 19, \mathrm{C} 20, \mathrm{C} 22, \mathrm{C} 24$.

${ }^{4} \mathrm{Sum}=\mathrm{C} 10: 1, \mathrm{C} 14: 1, \mathrm{C} 15: 1, \mathrm{C} 16: 1, \mathrm{C} 17: 1$, cis-9 C18:1, cis-11 C18:1, cis-12 C18:1, cis-9,cis-12 C18:2, cis9,cis-15 C18:2, cis-9,cis-12,cis-15 C18:3, C20:1n-9, C20:3n-3, C20:4n-6, C20:5n-3, C22:1n-9, C22:2, C22:5n-3, C22:6n-3, C24:1.

${ }^{5} \mathrm{Sum}=$ cis-9,cis-12 C18:2, cis-9,cis-15 C18:2, cis-9,cis-12,cis-15 C18:3, C20:3n-3, C20:4n-6, C20:5n-3, C22:2, C22:5n-3, C22:6n-3.

${ }^{6} \mathrm{Sum}=\mathrm{C} 10: 1, \mathrm{C} 14: 1, \mathrm{C} 16: 1, \mathrm{C} 17: 1$, cis-9 C18:1, cis-11 C18:1, cis-12 C18:1, C20:1n-9, C22:1n-9, C24:1. 
Table 2. Effect of nanoemulsified soybean oil-fish oil mix on total bacterial count, IVDMD, and the rumen fatty acid proportion (g/100 g of fatty acids)

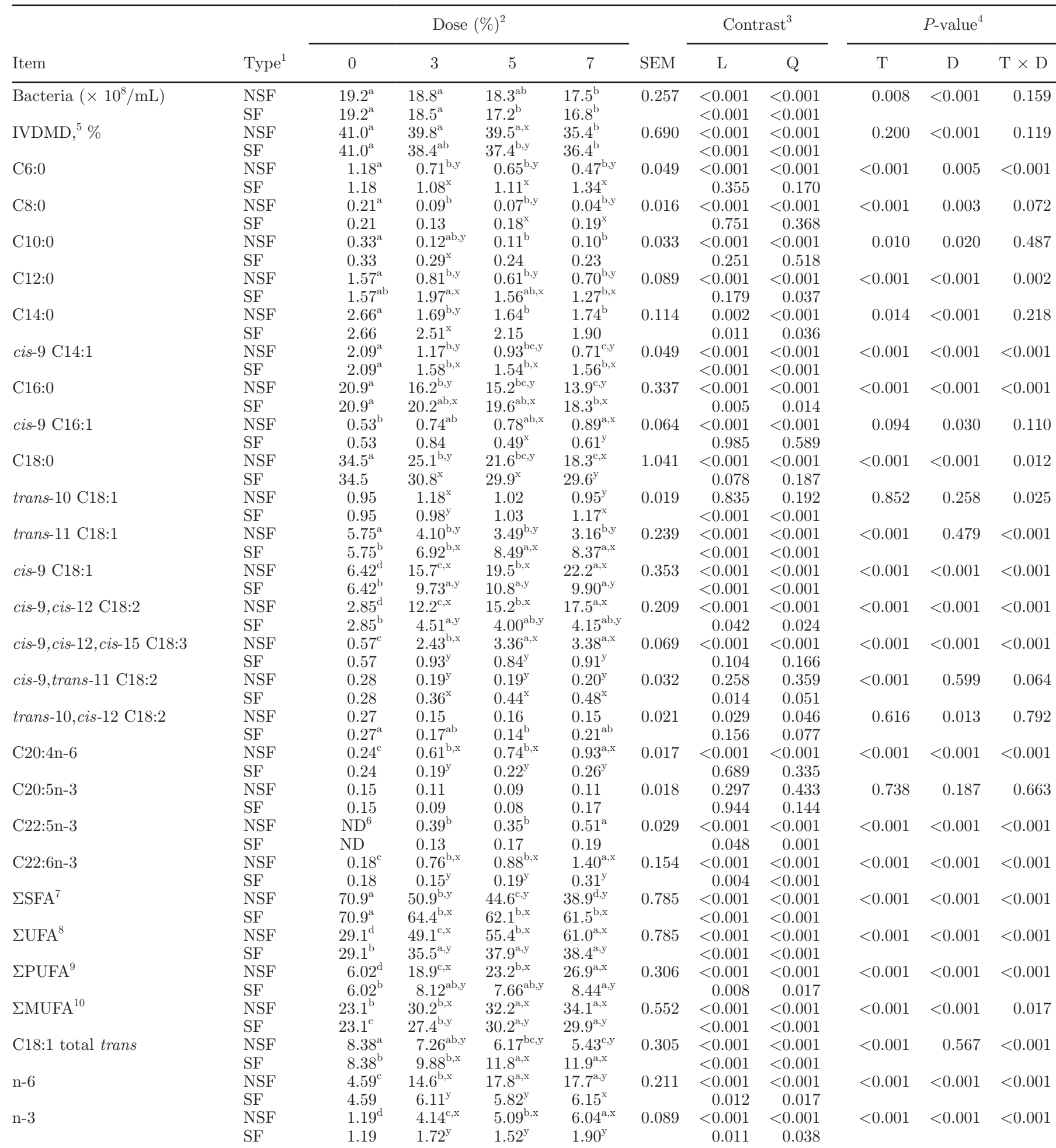


Table 2 (Continued). Effect of nanoemulsified soybean oil-fish oil mix on total bacterial count, IVDMD, and the rumen fatty acid proportion (g/100 g of fatty acids)

\begin{tabular}{|c|c|c|c|c|c|c|c|c|c|c|c|}
\hline \multirow[b]{2}{*}{ Item } & \multirow[b]{2}{*}{ Type $^{1}$} & \multicolumn{4}{|c|}{ Dose $(\%)^{2}$} & \multicolumn{3}{|c|}{ Contrast $^{3}$} & \multicolumn{3}{|c|}{$P$-value ${ }^{4}$} \\
\hline & & 0 & 3 & 5 & 7 & SEM & L & $\mathrm{Q}$ & $\mathrm{T}$ & $\mathrm{D}$ & $\mathrm{T} \times \mathrm{D}$ \\
\hline
\end{tabular}

\footnotetext{
${ }^{\mathrm{a}-\mathrm{d}}$ Means within a row with different superscripts differ $(P<0.05)$.

${ }^{\mathrm{x}, \mathrm{y}}$ Means within a column with different superscripts differ $(P<0.05)$.

${ }^{1}$ Type of supplements = nanoemulsified soybean:fish oil blend (NSF) and raw soybean:fish oil blend (SF).

${ }^{2}$ Doses of supplemented oils (\%).

${ }^{3}$ Contrasts: significance of linear (L) and quadratic (Q) components of the response to the supplemented levels of soybean:fish oil blend in both nanoemulsified and raw forms.

${ }^{4}$ Probability of significant effect due to the soybean oil:fish oil blend type $(\mathrm{T})$, dose $(\mathrm{D})$, and their interaction $(\mathrm{T} \times \mathrm{D})$.

${ }^{5}$ In vitro DM digestibility.

${ }^{6}$ Not detected.

${ }^{7} \mathrm{Sum}=\mathrm{C} 6, \mathrm{C} 8, \mathrm{C} 10, \mathrm{C} 12, \mathrm{C} 12$ iso, $\mathrm{C} 12$ anteiso, $\mathrm{C} 13$ iso, C14, C14 iso, C14 anteiso, C15, C15 iso, C16, C16 iso, C16 anteiso; C17, C17 iso, $\mathrm{C} 17$ anteiso, $\mathrm{C} 18, \mathrm{C} 19, \mathrm{C} 20, \mathrm{C} 22, \mathrm{C} 24$.

${ }^{8} \mathrm{Sum}=\mathrm{C} 10: 1, \mathrm{C} 14: 1, \mathrm{C} 15: 1, \mathrm{C} 16: 1, \mathrm{C} 17: 1$, trans-5 C18:1, trans-6-8 C18:1, trans-9 C8:1, trans-10 C18:1, trans-11 C18:1, trans-12 C18:1, trans-15 C18:1, cis-9 C18:1, cis-11 C18:1, cis-12 C18:1, cis-13 C18:1, cis-14 C18:1, cis-15 C18:1, trans-10,cis-12 C18:2, cis-9,trans-12 C18:2, cis9, cis-12 C18:2, cis-9, cis-15 C18:2, cis-9,cis-12,cis-15 C18:3, C20:1n-9, C20:3n-3, C20:4n-6, C20:5n-3, C22:1n-9, C22:2, C22:5n-3, C22:6n-3, C24:1. ${ }^{9} \mathrm{Sum}=$ trans-10,cis-12 C18:2, cis-9,trans-12 C18:2, cis-9,cis-12 C18:2, cis-9, cis-15 C18:2, cis-9,cis-12,cis-15 C18:3, C20:3n-3, C20:4n-6, C20:5n-3, $\mathrm{C} 22: 2, \mathrm{C} 22: 5 \mathrm{n}-3, \mathrm{C} 22: 6 \mathrm{n}-3$.

${ }^{10} \mathrm{Sum}=\mathrm{C} 10: 1, \mathrm{C} 14: 1, \mathrm{C} 15: 1, \mathrm{C} 16: 1, \mathrm{C} 17: 1$, trans-5 C18:1, trans-6-8 C18:1, trans-9 C18:1, trans-10 C18:1, trans-11 C18:1, trans-12 C18:1, trans-15 C18:1, cis-9 C18:1, cis-11 C18:1, cis-12 C18:1, cis-13 C18:1, cis-14 C18:1, cis-15 C18:1, C20:1n-9, C22:1n-9, C24:1.
}

the slight difference in fatty acid proportions of the produced nanoemulsion compared with that in raw oil.

The effects of the soybean-fish oil blend in both nanoemulsified (NSF) and raw forms (SF) on the total bacterial count, IVDMD, and fatty acid proportions in the fermentation fluid samples are presented in Table 2. Total bacterial count was negatively affected by the SF levels of supplementation. As the amount of SF supplement increased, a quadratic reduction in the total bacterial count in the fermentation fluid $(P$ $<0.001$ ) was observed. The decrease in the bacterial count was followed by a quadratic decrease $(P<0.001)$ in IVDMD; these decreases in total bacterial counts and IVDMD were greatest with the highest levels of SF addition $(5$ and $7 \%)$. Quadratic reductions $(P<0.001)$ in total bacterial count and IVDMD were also observed for the NSF form of addition. Supplementation with SF led to quadratic increases $(P<0.001)$ in the proportion of trans-11 C18:1 (vaccenic acid, VA). In contrast, supplementation with an increasing level of NSF resulted in a quadratic reduction $(P<0.001)$ in the proportion of VA. Similarly, the proportion of cis-9,trans-11 C18:2 tended to increase linearly $(P<0.05)$ with SF supplementation. Supplementing the rapeseed-fish oil blend in raw form (RF; Table 3) led to quadratic reductions in total bacterial count $(P<0.001)$. This decline in total bacterial count was connected to a quadratic reduction $(P<0.001)$ in IVDMD. For the NRF blend, the results matched those of NSF, such that an increased level of $\mathrm{NRF}$ addition led to a quadratic reduction $(P<0.001)$ in bacterial count and IVDMD. Both RF and NRF supplementation led to trends similar to those observed when SF and NSF were added, regarding the proportions of VA and cis-9,trans-11 C18:2. A quadratic increase in VA $(P<0.001)$ was observed as the levels of RF supplementation increased, and proportions of cis-9,trans-11 C18:2 increased linearly $(P<0.001)$ with $\mathrm{RF}$ addition. Supplementation with NRF quadratically reduced $(P<0.05)$ the proportion of VA; however, despite the decrease in VA by NRF, a similar linear effect $(P>0.20)$ of NRF levels on the cis-9,trans-11 C18:2 proportion was observed.

The raw forms of the oil blends rich in C18 UFA included in both batch experiments led to a shift in the fatty acid proportions compared with the other treatments. These results showed that the biohydrogenation that occurred in the batch cultures matched the normal condition in the rumen. Previous studies have demonstrated an increase in VA and CLA outflow from the rumen when dairy cows were fed plant oils rich in C18 UFA (Boerman and Lock, 2014) or fish oil (AbuGhazaleh and Ishlak, 2014). Increasing the proportion of cis-9,trans-11 C18:2 in rumen and ruminant product is, at this stage, mainly limited by the amount of VA outflow from the rumen. Supplementing ruminant diets with fish oil is an efficient way of accumulating VA. 
Table 3. Effect of nanoemulsified rapeseed oil:fish oil mixes on total bacterial count, IVDMD, and rumen fatty acids proportion ( $\mathrm{g} / 100 \mathrm{~g}$ of fatty acids)

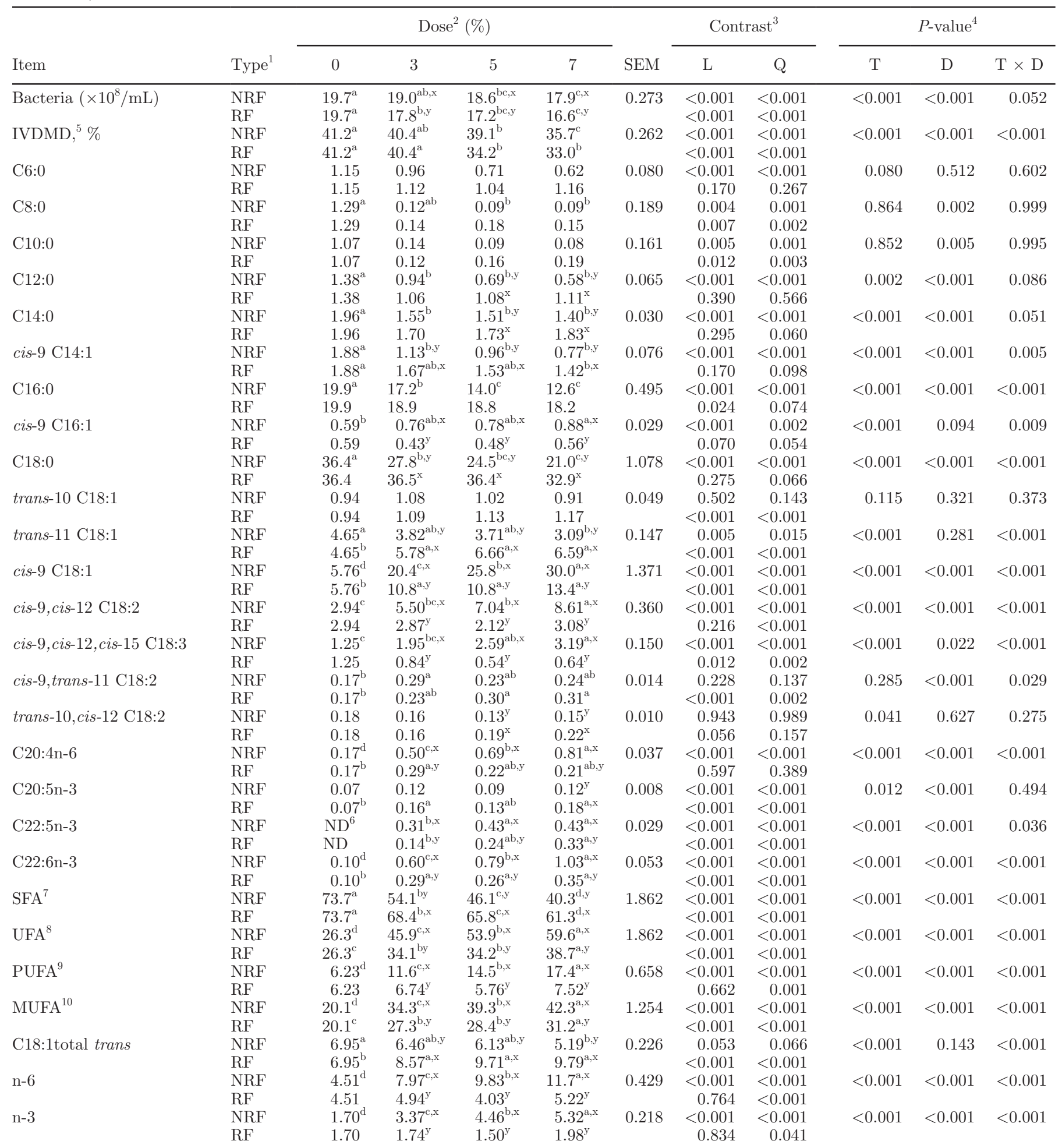


obtained by NSF addition. A quadratic elevation $(P<$ $0.001)$ in PUFA proportion and a quadratic reduction $(P<0.001)$ in SFA was observed with NSF levels that decreased PUFA in the fermentation culture to a lesser extent. The same trend was observed when using the RF oil blends (Table 3). We found that NRF levels led to quadratic elevations of proportions of oleic acid, linoleic acid, $\alpha$-linolenic acid, EPA, and DHA $(P<$ 0.001). In the case of RF addition, quadratic reductions $(P<0.05)$ were observed in proportions of oleic acid, EPA, and DHA. Increasing addition of RF resulted in a quadratic elevation $(P<0.001)$ in linoleic acid proportions. However, when RF levels were contrasted against the control treatment diet, the linear proportion of linoleic acid was similar to levels of RF addition. Quadratic reductions in the proportion of $\alpha$-linolenic acid $(P<0.05)$ were observed with increasing levels of RF. All levels of the nanoemulsified RF supplementation led to quadratic reductions $(P<0.001)$ in the proportion of SFA and quadratic elevations $(P=0.001)$ in the proportion of PUFA in the fermentation fluid samples. The same observations were made for NRF addition, which resulted in quadratic elevations $(P<0.001)$ in the PUFA proportions and quadratic reductions $(P<$ 0.001) in the SFA proportions.

Despite past evidence that ruminal biohydrogenation is a complex biochemical process involving a wide range of fatty acid intermediates, the published pathways for linoleic acid BH have changed over the decades. A delay in the biohydrogenation of linoleic acid compared with $\alpha$-linolenic acid has been shown, which could be due to the preferential uptake of $\alpha$-linolenic acid by rumen bacteria or to differences in microbial isomerase or saturase affinity between linoleic acid and $\alpha$-linolenic acid (Martínez Marín et al., 2013). Additionally, the release rate of fatty acids may vary considerably depending on the source and form of the added lipids (Dohme et al., 2003). The wide range of data and literature highlights the role of rumen microorganisms in C18 UFA biohydrogenation. What is not well documented is whether the same pathways apply to EPA and DHA. It has been suggested that the more unsaturated the fatty acids are, the greater the effect on the activity of rumen bacterial and protozoan populations. The great effect on the level of VA produced and accumulated in the rumen, especially by DHA supplementation, would then be explained (Klein and Jenkins, 2011). It seems that the nanoemulsion technology helps to preserve higher proportions of PUFA compared with the raw addition. This could be due to the direct inhibition of ruminal lipolysis or biohydrogenation, which consequently preserves a high proportion of PUFA from being saturated under biohydrogenation condition.
In conclusion, the observed favorable increase in in vitro rumen UFA (especially n-3 and n-6) when nanoemulsified oil blend forms were supplemented suggests that nanoscale droplets of supplemented PUFA could be effective in preserving higher proportions of PUFA, which would thus be available for absorption regardless of the type of oil used. Oil-in-water nanoemulsions may represent a promising, effective strategy for the supplementation of unsaturated lipids, which could be mixed with fresh drinking water for dairy cows. However, further research is needed to clarify the mode of action of nanoscale oil droplets on rumen microorganisms.

\section{ACKNOWLEDGMENTS}

The present experiment was financed by the Poznan University of Life Sciences research grants for young researchers, projects number 507.533.13 and 507.533.42. The study scheme was a part of M. El-Sherbiny general mission $(\mathrm{PhD})$ proposal financed by the Cultural Affairs and Missions Sectors, Ministry of Higher Education, Egypt.

\section{REFERENCES}

AbuGhazaleh, A. A., and A. Ishlak. 2014. Effects of incremental amounts of fish oil on trans fatty acids and Butyrivibrio bacteria in continuous culture fermenters. J. Anim. Physiol. Anim. Nutr. (Berl.) 98:271-278.

Ahmadi Lakalayeh, G., R. Faridi-Majidi, R. Saber, A. Partoazar, S. E. Mehr, and A. Amani. 2012. Investigating the parameters affecting the stability of superparamagnetic iron oxide-loaded nanoemulsion using artificial neural networks. AAPS PharmSciTech 13:1386-1395.

Boerman, J. P., and A. L. Lock. 2014. Effect of unsaturated fatty acids and triglycerides from soybeans on milk fat synthesis and biohydrogenation intermediates in dairy cattle. J. Dairy Sci. 97:7031-7042.

Cieslak, A., A. Machmüller, M. Szumacher-Strabel, and M. R. L. Scheeder. 2009. A comparison of two extraction methods used to quantify the $\mathrm{C} 18$ fatty acids in feed and digesta of ruminants. J. Anim. Feed Sci. 18:362-367.

Cieslak, A., P. Zmora, A. Stochmal, L. Pecio, W. Oleszek, E. PersKamczyc, J. Szczechowiak, A. Nowak, and M. Szumacher-Strabel. 2014. Rumen antimethanogenic effect of Saponaria officinalis L. phytochemicals in vitro. J. Agric. Sci. 152:981-993.

Dohme, F., V. Fievez, K. Raes, and D. I. Demeyer. 2003. Increasing levels of two different fish oils lower ruminal biohydrogenation of eicosapentaenoic and docosahexaenoic acid in vitro. Anim. Res. 52:309-320.

Ericsson, M., D. Hanstorp, P. Hagberg, J. Enger, and T. Nystrom. 2000. Sorting out bacterial viability with optical tweezers. J. Bacteriol. 182:5551-5555.

Fathi, M., M. R. Mozafari, and M. Mohebbi. 2012. Nanoencapsulation of food ingredients using lipid based delivery systems. Trends Food Sci. Technol. 23:13-27.

Ghosh, V., A. Mukherjee, and N. Chandrasekaran. 2014. Eugenolloaded antimicrobial nanoemulsion preserves fruit juice against, microbial spoilage. Colloids Surf. B Biointerfaces 114:392-397.

Ishlak, A., A. A. AbuGhazaleh, and M. Günal. 2014. Short communication: Effect of blackberry and pomegranate oils on vaccenic acid formation in a single-flow continuous culture fermentation system. J. Dairy Sci. 97:1067-1071. 
Kentish, S., T. J. Wooster, M. Ashokkumar, S. Balachandran, R. Mawson, and L. Simons. 2008. The use of ultrasonics for nanoemulsion preparation. Innov. Food Sci. Emerg. Technol. 9:170-175.

Khiaosa-ard, R., F. Leiber, and C. R. Soliva. 2010. Methods of emulsifying linoleic acid in biohydrogenation studies in vitro may bias the resulting fatty acid profiles. Lipids 45:651-657.

Klein, C. M., and T. C. Jenkins. 2011. Docosahexaenoic acid elevates trans-18:1 isomers but is not directly converted into trans-18:1 isomers in ruminal batch cultures. J. Dairy Sci. 94:4676-4683.

Koba, K., and T. Yanagita. 2014. Health benefits of conjugated linoleic acid (CLA). Obes. Res. Clin. Pract. 8:e525-e532.

Martínez Marín, A. L., P. Gomez-Cortes, G. Gomez Castro, M. Juarez, L. Perez Alba, M. Perez Hernandez, and M. A. de la Fuente. 2013. Time-dependent variations in milk fatty acid content of goats fed 3 different plant oils. J. Dairy Sci. 96:3238-3246.
Mason, T. G., J. N. Wilking, K. Meleson, C. B. Chang, and S. M. Graves. 2006. Nanoemulsions: Formation, structure, and physical properties. J. Phys. Condens. Matter 18:635-666.

McCrorie, T. A., E. M. Keaveney, J. M. W. Wallace, N. Binns, and M. B. E. Livingstone. 2011. Human health effects of conjugated linoleic acid from milk and supplements. Nutr. Res. Rev. 24:206-227.

Szumacher-Strabel, M., S. A. Martin, A. Potkanski, A. Cieslak, and J. Kowalczyk. 2004. Changes in fermentation processes as the effect of vegetable oil supplementation in in vitro studies. J. Anim. Feed Sci. 13:215-218.

Zhang, Z., F. Vriesekoop, Q. Yuan, and H. Liang. 2014. Effects of nisin on the antimicrobial activity of D-limonene and its nanoemulsion. Food Chem. 150:307-312. 